\title{
EFEITOS DO TREINAMENTO AERÓBICO (TA) E DO TREINAMENTO RESISTIDO (TR) NA COMPOSIÇÃO CORPORAL
}

\section{ARTIGO DE REVISÃO}

SOUZA, Eliene Lopes de ${ }^{1}$

CARVALHO, Crisley Cássia Barbosa de ${ }^{2}$

SILVA, Sebastião Lobo da ${ }^{3}$

SANTOS, Givanildo de Oliveira ${ }^{4}$

SOUZA, Eliene Lopes de. Et al. Efeitos do Treinamento Aeróbico (TA) e do Treinamento Resistido (TR) na composição corporal. Revista Científica Multidisciplinar Núcleo do Conhecimento. Ano 05, Ed. 09, Vol. 03, pp. 131-143. Setembro de 2020. ISSN: 2448-0959, Link de acesso: https://www.nucleodoconhecimento.com.br/educacao-fisica/treinamentoaerobico

\section{RESUMO}

Os benefícios dos exercícios físicos regulares estão bem documentados, mas às recomendações do modo de exercício para benefícios específicos à saúde permanece incerta, em grande parte devido aos escassos dados científicos que apoiam essas recomendações. Objetivou-se verificar em artigos científicos os benefícios e efeitos do treinamento aeróbico e treinamento resistido em praticantes com sobrepeso e obesos. Os estudos demonstraram que o treinamento aeróbico, treinamento de

${ }^{1}$ Bacharel em Educação Física, pela Faculdade de Piracanjuba - GO.

${ }^{2}$ Bacharel em Educação Física, pela Faculdade de Piracanjuba - GO.

${ }^{3}$ Doutorando em Educação Física, pela Universidade Católica de Brasília - DF.

${ }^{4}$ Mestre em Tecnologia de Alimentos, pelo Instituto Federal Goiano - Campus Rio Verde - GO. 
resistência, ou a combinação entre eles, diminuem o porcentual de gordura corporal em adolescentes e adultos com sobrepesos e obesos. O treinamento aeróbico: melhora no sistema oxidativo dependente de metabolismo metabólico, capacidade metabólica e cardiorrespiratória. Já os praticantes do treinamento resistido aumentam a massa muscular, melhor a densidade do músculo esquelético; melhora o diâmetro da fibra muscular e força muscular.

Palavras-chave: Exercícios Físicos, obesidade, saúde, musculação, treinamento concorrente.

\section{INTRODUÇÃO}

Os benefícios dos exercícios físicos regulares estão bem documentados, mas às recomendações do modo de exercício para benefícios específicos à saúde permanece incerta, em grande parte devido aos escassos dados científicos que apoiam essas recomendações. Existem dados que aproximadamente dois terços dos adultos que estão com sobrepeso ou obesidade, os médicos exigem diretrizes claras de exercícios com base em evidências científicas suficientes para prescrever o plano de exercícios se tornando com maior eficácia (OGDEN et al., 2004).

Embora muitos profissionais tenham focado em orientações sobre exercícios de resistência ou treinamento aeróbico para perda e manutenção de peso, algumas diretrizes e declarações de posição voltadas para a redução e manutenção do peso corporal sugeriram que o treinamento resistido também pode ser eficaz para redução de gordura corporal (DONNELLY et al., 2009).

Em alguns casos, as diretrizes podem levar a percepções errôneas entre alguns profissionais do exercício e leigos sobre a força das evidências e a eficácia do Treinamento Resistido (TR) na eficácia de perda de peso e gordura corporal, levando as pessoas a acreditar que somente o Treinamento Aeróbico (TA) demonstraram a redução da gordura corporal (OLSON et al., 2007). 
As diretrizes para exercícios devem basear-se em evidências inequívocas de relações específicas entre o modo de exercício e as mudanças na massa corporal e na gordura. É interessante notar que, apesar da prevalência da obesidade e da posição múltipla existente promovendo exercícios para o tratamento da obesidade, existem poucos estudos que compararam diretamente os efeitos da TA do TR ou de uma combinação das duas (TA / RT) na massa gorda em adultos com sobrepeso e obesidade (OLSON et al., 2007).

Os estudos existentes não estudaram diretamente quantidades comparáveis de Treinamento Aeróbico (TA) e Treinamento Resistido (TR). Portanto, resta determinar se uma quantidade significativa de TR diminuirá a massa gorda em adultos com sobrepeso e obesidade, se TA ou TR é mais eficaz na redução da massa gorda quando a exposição (tempo) é mantida constante e se uma combinação de treinamento aeróbico e de resistência (TA / TR) fornece melhorias aditivas na composição corporal.

Conclui-se que embora tenha sido mais eficaz para ganhos de massa corporal magra, a TR e reduzi significativamente a massa gorda ou a massa corporal total. O TA é mais eficaz que o TR na redução de gordura e massa corporal em adultos previamente sedentários. Embora exigi o dobro de tempo, um programa combinado de TA e TR resulta em maior perda de massa gorda ou massa corporal do que em TA.

Se o objetivo é aumentar a massa e a força muscular, é necessário um programa que inclua TR. No entanto, equilibrando os compromissos de tempo com os benefícios de saúde acumulados, parece que o TA sozinho é o modo ideal de exercício para reduzir a massa gorda e a massa corporal total. 


\section{DESENVOLVIMENTO}

\section{METODOLOGIA}

Os levantamentos dos dados foram realizados nas plataformas, Pubmed, Science Direct, e Google scholar. Utilizou os seguintes termos para pesquisa: treinamento aeróbico (TA), treinamento resistido (TR) e combinação de TA e TR.

Incluindo publicações ou artigos voltados para adolescentes, adultos com sobrepeso, obesidade, nas publicações selecionadas, os seguintes dados: Ano de estudo; tamanho da amostra e qualidade da revista publicada.

\section{TREINAMENTO RESISTIDO}

O treinamento resistido é eficaz para aprimorar vários aspectos importantes da saúde física e mental. Começando com a redução progressiva da massa muscular e metabolismo de repouso associados ao envelhecimento inativo, os estudos de treinamento de resistência demonstraram consistentemente aumentos significativos no peso magro e na taxa metabólica, acompanhados por diminuições significativas no peso da gordura (DONNELLY et al., 2009).

Nas várias áreas que envolvem desempenho físico, o treinamento resistido tem sido associado à redução da dor lombar, diminuição do desconforto artrítico, maior independência funcional, maior controle do movimento e maior velocidade de caminhada. Com base em inúmeros estudos que mostraram melhora da homeostase da glicose e insulina, o treinamento resistido foi recomendado para resistir ao diabetes tipo 2 (STRASSER et al., 2010).

Na saúde cardiovascular, as pesquisas de treinamento de resistência demonstraram redução da pressão arterial em repouso, melhor perfil lipídico no sangue e melhora da condição vascular. $O$ treinamento resistido parece ter maior impacto na densidade óssea (DMO) do que outros tipos de atividade física e demonstrou aumentar significativamente a DMO em adultos de todas as idades (BANZ et al., 2003). 
Os benefícios demonstrados para a saúde mental do treinamento de resistência incluíram diminuição dos sintomas de depressão, aumento da autoestima e autoconceito físico e melhora da capacidade cognitiva. Finalmente e fundamentalmente, o treinamento resistido demonstrou reverter os fatores de envelhecimento no músculo esquelético (DONNELLY et al., 2009).

A prescrição do exercício de TR utilizada em estudo representa o limite superior da quantidade recomendada pelo American College of Sports Medicine em termos de sessões por semana e número de séries por sessão (WHALEY; BRUBAKER; OTTO, 2006). A TR induziu ganhos significativos na massa corporal magra e na força, a falta de perda de massa corporal observada com a TR neste estudo apoia as pessoas que é motivada por um aumento na massa corporal magra (DONNELLY et al., 2009).

No entanto, existem relatos conflitantes na literatura sobre se a TR induzir ou não a perda de massa gorda: alguns ensaios clínicos randomizados descobrem que a TR reduziu significativamente a massa gorda, enquanto outros relatam uma tendência estatisticamente insignificante ou nenhuma alteração na massa gorda (DAVIDSON et al., 2009). No entanto, deve-se enfatizar que a TR melhorou significativamente a massa corporal magra, conforme confirmado por medições da área muscular da coxa.

Embora alguns autores afirmam o efeito do TR na massa gorda é inconclusiva e que o treinamento resistido não é eficaz para perda de peso, a TR ainda é endossada como um meio eficaz para o tratamento da obesidade. Da mesma forma, outros documentos de consenso e relatórios de estudos incluem tabelas que mostram que a TR resulta em diminuição do percentual de gordura, com a sugestão de que essa redução no percentual de gordura indica uma diminuição na massa gorda (BANZ et al., 2003).

O problema em relatar alterações no percentual de gordura, em vez da massa gorda absoluta, foi demonstrado pelo grupo de TR em estudo randomizado, o qual o percentual de gordura diminuiu significativamente sem nenhuma alteração na massa gorda absoluta. Em outras palavras, as alterações na porcentagem de gordura corporal foram motivadas apenas pelo aumento da massa corporal magra induzida 
pela TR, mesmo com um programa de treinamento de resistência bastante substancial, com duração de 8 meses (LEMMER et al., 2001).

Talvez a razão mais citada para a redução da massa gorda e do peso corporal pela TR seja que a taxa metabólica de repouso (RMR) aumenta teoricamente à medida que a massa corporal magra aumenta, resultando em um aumento no gasto energético total e uma mudança negativa correspondente no balanço energético (LEMMER et al., 2001).

Foram observados em estudos que o TR aumentou a massa corporal magra sem uma mudança significativa na massa gorda ou no peso corporal. Essas observações, juntamente com as de outros estudos, talvez seja hora de reconsiderar seriamente a opinião convencional de que a TR sozinha pode induzir alterações na massa corporal ou na gordura devido a um aumento no metabolismo de adultos sedentários com sobrepeso ou obesidade (DAVIDSON et al., 2009).

À medida que o problema da obesidade aumenta, aumenta também a prevalência de diabetes tipo 2. Prevê-se que, em meados deste século, um dos três adultos tenha diabetes (BOYLE, 2010). Em seu artigo de revisão sobre envelhecimento, treinamento resistido e prevenção de diabetes, concluíram que o treinamento resistido pode ser uma abordagem de intervenção eficaz para adultos de meia idade e idosos para combater os declínios associados à idade na sensibilidade à insulina e prevenir o aparecimento de diabetes tipo 2 (FLACK K et al., 2011).

Conforme foram demonstrados que o treinamento resistido reduz a gordura abdominal, o que pode ser particularmente importante para a prevenção do diabetes. Isso ocorre porque a resistência à insulina parece estar associada ao acúmulo de gordura abdominal em adultos idosos (KOHRT et al., 1993).

Com base em sua revisão de literatura, sugeriram que programas de treinamento de resistência que incorporam protocolos de maior volume e maior intensidade podem ser mais eficazes para melhorar a resistência à insulina e a tolerância à glicose em 
comparação com os protocolos de exercícios de menor volume e menor intensidade (FLACK et al., 2011).

Uma metanálise revelou que o treinamento resistido reduziu o tecido adiposo visceral e diminuiu a hemoglobina glicosilada em pessoas com metabolismo anormal da glicose. Os autores da revisão concluíram que o treinamento resistido deve ser recomendado para a prevenção e tratamento do diabetes tipo 2 e distúrbios metabólicos (STRASSER et al., 2010).

O treinamento resistido está associado à melhora da homeostase da glicose e da insulina devido a aumentos na área da seção transversal muscular e na massa corporal magra, além de melhorias qualitativas nas propriedades metabólicas musculares, incluindo aumentos na densidade do transportador de glicose, atividade de glicogênio sintase e depuração de glicose mediada por insulina. Também existem evidências de que o treinamento resistido pode ser preferível ao exercício aeróbico para melhorar a sensibilidade à insulina e diminuir a hemoglobina glicosilada (BWEIR et al., 2009).

\section{TREINAMENTO AERÓBICO}

O treinamento aeróbico (TA) aumenta o pico de consumo de oxigênio (VO2 Máx), que está intimamente relacionado ao percentual total de gordura corporal (\% GC); o (TA) também é uma estratégia poderosa para perda de peso, particularmente perda de gordura corporal (ODA et al., 2014).

Ao projetar um programa de perda de peso adequado, a duração e a intensidade do exercício geralmente são manipuladas. O treinamento aeróbico moderado de 150 minutos por semana, poderá melhorar os fatores de risco para síndrome metabólica, como composição corporal, resistência à insulina e hemoglobina glicada (O'HAGAN et al., 2013).

O American College of Sports Medicine sugeri que treinamento aeróbico moderado a longo prazo, sendo maior que 150 ou 300 min por semana podem reduzir 
significativamente o peso corporal quando a dieta não é controlada (DONNELLY et al., 2010).

No entanto, quando as intensidades dos exercícios diferem, o gasto energético com exercícios não é o único fator responsável pela perda de peso (WILLIAMS et al., 2013). Os efeitos do aumento da intensidade do exercício na perda de peso quando a duração do exercício é mantida constante permanecem desconhecidos.

O treinamento físico de alta intensidade pode reduzir efetivamente a gordura corporal e abdominal (LEE et al., 2012). Quando o gasto energético é mantido igual, o exercício de alta intensidade é mais benéfico para melhorar a composição corporal e reduzir a gordura abdominal do que o exercício de baixa intensidade (LEE et al., 2012).

Em um estudo foi possível relatar que o treinamento intervalado de alta intensidade, foi igualmente eficaz na redução do peso corporal e da gordura corporal, assim como o treinamento de resistência de baixa intensidade (TJONNA et al., 2008).

Essas evidências sugerem que a maior intensidade de exercício pode ser mais eficaz na melhoria da composição corporal. No entanto, evidências que o efeito do exercício com diferentes intensidades e gasto de energia na composição corporal são escassas ao longo prazo. Além disso, o uso de uma única duração durante o treinamento com exercícios aeróbicos pode aumentar o apetite das pessoas com obesidade durante o período de recuperação (GEORGE; MORGANSTEIN, 2003).

\section{TREINAMENTO AERÓBICO VERSUS TREINAMENTO DE RESISTÊNCIA}

É importante que os profissionais entendam que o treinamento aeróbico ou de resistência são necessários nas alterações da composição corporal em geral. Comparações entre grupos TA e TR em estudos sugerem que o TA diminui significativamente o peso corporal e a massa gorda significativamente mais do que 0 TR. Enquanto que a combinação destes, produziram mudanças estatisticamente semelhantes no percentual de gordura corporal, essas alterações foram 
impulsionadas por diferentes mecanismos, nos quais a RT aumentou a massa corporal magra e a TA diminuiu a massa gorda (BATEMAN et al., 2011).

Estes dados são corroborados por estudos que indicam que o TA reduzi significativamente o tecido adiposo visceral mais do que o TR e tendeu para o mesmo resultado na alteração da gordura hepática (BATEMAN et al., 2011). Além disso, uma metanálise dos efeitos do treinamento aeróbico versus do treinamento de resistência sobre a gordura visceral conclui que há uma tendência em direção a maior redução na gordura visceral com o TA quando comparado ao TR (ISMAIL et al., 2012).

Estes dados, demonstram que os conhecimentos em programa de TR no TA são equivalentes, fornecendo evidências convincentes de que o TA é o modo ideal de exercício para melhorar a quantidade de gordura corporal (ANDERSEN; JAKICIC, 2009).

Encontramos poucos estudos que examinaram os efeitos de TA e RT em adultos com sobrepeso ou obesidade. Foram examinados em TA e TR, e uma combinação dos dois em uma população diabética (SIGAL et al., 2007). Foi possível observar a redução significativa na massa corporal e na gordura com TA e uma tendência à diminuição da massa gorda com TR, mas o TR não produziu alteração na massa corporal em comparação com os grupos controles. Embora intuitivamente esses resultados sugiram que o TA seja mais eficaz que o TR, nenhuma comparação direta foi feita entre TA e TR na análise estatística e, portanto, nenhuma conclusão definitiva poderá ser feita entre os dois modos de exercício neste estudo (SIGAL et al., 2007).

Foram analisados o TA e TR e a combinação dos dois, onde o grupo TR realizou apenas um conjunto de treinamento, três vezes por semana, durante o total de 60 minutos de treinamento por semana, enquanto o grupo TA exercitou-se por 150 minutos por semana. A diferença de tempo entre os grupos no estudo, limita a comparação da eficácia dos dois modos de exercício na composição corporal (DAVIDSON et al., 2009). 
O tempo gasto no exercício não equivale ao gasto energético do exercício realizado, pois o tempo de TR inclui os tempos gastos na recuperação entre os conjuntos e na movimentação entre as várias máquinas. No entanto, comparações baseadas no tempo total gasto no exercício ajudam a esclarecer qual modo de exercício é mais eficiente para afetar as variáveis de interesse, levando em consideração aos resultados, o TA foi o método de exercício mais eficiente para mudanças favoráveis na gordura corporal (DAVIDSON et al., 2009).

Foram realizados TA comparados ao TR a um programa de TA, proporcionando benefícios adicionais para a redução da massa gorda. Um comunicado de 2000 da American Heart Association Science, afirma que a RT complementa a TA para controle de peso (POLLOCK et al., 2000). Em estudo, encontraram que a combinação de TA e TR melhoraram significativamente a massa gorda do que TA isoladamente em diabéticos tipo II (CHURCH et al., 2010).

No entanto, as observações nestes protocolos de treinos TA e TR sugerem que a adição de TR não melhorou significativamente os resultados do grupo TA na indução de controle de peso em um grupo diabético, que não praticava exercício físico, com sobrepeso e meia idade. Talvez as diferenças demográficas entre os dois estudos ofereçam uma explicação para as diferentes conclusões. Os resultados do TA e TR são apoiados no relatório citado anteriormente examinando qual método de exercício é mais eficaz quando o tempo é mantido constante em uma população não diabética (BATEMAN et al., 2011).

Em grupos TA e TA combinado com o TR exercitado - se por 150 min por semana e foram significativamente melhores na redução de massa corporal e gordura do que 0 grupo TR, mas não foram significativamente diferentes entre si. Enquanto foram observados que o exercício combinado de TA com TR como ideal para melhorar as limitações funcionais, isso não era foi igual para a redução da massa gorda corporal. $O$ estudo reforça esta observação, pois foram observadas perdas semelhantes de peso corporal e massa gorda nos grupos TA e TA com TR, embora a duração do exercício no grupo combinado fosse aproximadamente o dobro da do grupo TA (BATEMAN et al., 2011). 


\section{EFEITOS NA COMPOSIÇÃO CORPORAL}

As diretrizes de atividade física recomendam a combinação de Treinamento Resistido (TR) e Treinamento Aeróbico (TA), para otimização da saúde e benefícios cardiovasculares, o American College of Sports Medicine confirmou que há faltas de evidências para indicar que a TR, sem TA ou controle dietético, é suficiente para promover a perda de peso e, portanto, recomenda-se a utilização em combinação. Em estudo de metanálise sugeriram que os exercícios simultâneos são realmente mais eficientes para parâmetros antropométricos, reduzindo o Índice de Massa Corporal (IMC) e a Massa Gorda (MG), bem como para aumentar a Taxa Metabólica Basal, quando comparado a um único tipo de exercício (DONNELLY et al., 2009).

De acordo com outras revisões sistemáticas em jovens obesos, os exercícios concorrentes promovem melhores resultados na composição corporal quando comparado apenas com TR e TA, os resultados são semelhantes relatados em estudos realizados em adultos com sobrepeso e obesos, demonstrando e evidenciando que os exercícios combinados se demonstraram mais eficaz na redução da obesidade (SCHWINGSHACKL et al., 2013). Além disso, estudos em meninos obesos e meninas demonstraram separadamente que o treinamento combinado (12 a 20 semanas) favorece a redução de $\pm 3 \%$ na adiposidade corporal (WONG et al., 2008).

Uma metanálise relatou a redução semelhante, porém de menor percentual, da adiposidade entre jovens obesos que praticava exercícios físicos comparados a quem não exercitam (1,93\% no percentual de massa gorda) (GARCíA et al., 2016).

No entanto, o treinamento físico concorrente, com intervenções prolongadas, poderão ser a prescrição ideal para maximizar os benefícios na adiposidade corporal e preservar a diminuição da massa muscular associada à perda de peso provocada por (TA) ou falta de exercício físico (CARNIER et al., 2013). 


\section{CONSIDERAÇÕES FINAIS}

Os estudos demonstraram que o treinamento aeróbico, treinamento de resistência, ou a combinação entre eles, diminuem o porcentual de gordura corporal em adolescentes e adultos com sobrepesos e obesos.

Em praticantes de treinamento combinado de treinamento aeróbicos e de resistência, ou um treinamento aeróbico diminui a gordura corporal, circunferência da cintura e IMC. E os praticantes de treinamento resistido, melhoram: aumentam o músculo esquelético; absorve o diâmetro da fibra; força muscular.

Os exercícios dessas variáveis deve ser executado em ambos treinamentos aeróbicos e de resistência, os benefícios significativos podem ser alcançados através de qualquer tipo de exercício, porém, a combinação entre os métodos de treinos potencializa os resultados na massa corporal magra e tecido adiposo.

\section{REFERÊNCIAS}

ANDERSEN, Ross E.; JAKICIC, John M. Interpreting the physical activity guidelines for health and weight management. Journal of Physical Activity and Health, v. 6, n. 5, p. 651-656, 2009.

BANZ, William J. et al. Effects of resistance versus aerobic training on coronary artery disease risk factors. Experimental Biology and Medicine, v. 228, n. 4, p. 434-440, 2003.

BATEMAN, Lori A. et al. Comparison of aerobic versus resistance exercise training effects on metabolic syndrome (from the Studies of a Targeted Risk Reduction Intervention Through Defined Exercise-STRRIDE-AT/RT). The American journal of cardiology, v. 108, n. 6, p. 838-844, 2011.

BOYLE, James P. et al. Projection of the year 2050 burden of diabetes in the US adult population: dynamic modeling of incidence, mortality, and prediabetes prevalence. Population health metrics, v. 8, n. 1, p. 29, 2010. 
BWEIR, Salameh et al. Resistance exercise training lowers HbA1c more than aerobic training in adults with type 2 diabetes. Diabetology \& metabolic syndrome, v. $1, \mathrm{n}$. 1, p. 27, 2009.

CARNIER, June et al. Aerobic training (AT) is more effective than aerobic plus resistance training $(A T+R T)$ to improve anorexigenic/orexigenic factors in obese adolescents. Appetite, v. 69, p. 168-173, 2013.

$\mathrm{CHURCH}$, Timothy S. et al. Effects of aerobic and resistance training on hemoglobin A1c levels in patients with type 2 diabetes: a randomized controlled trial. Jama, v. 304, n. 20, p. 2253-2262, 2010.

DAVIDSON, Lance E. et al. Effects of exercise modality on insulin resistance and functional limitation in older adults: a randomized controlled trial. Archives of internal medicine, v. 169, n. 2, p. 122-131, 2009.

DONNELLY, Joseph E. et al. Appropriate physical activity intervention strategies for weight loss and prevention of weight regain for adults. Medicine \& Science in Sports \& Exercise, v. 41, n. 2, p. 459-471, 2009.

FLACK, Kyle D. et al. Aging, resistance training, and diabetes prevention. Journal of aging research, v. 2011, 2011.

GARCÍA-HERMOSO, Antonio et al. Exercise-based interventions and C-reactive protein in overweight and obese youths: a meta-analysis of randomized controlled trials. Pediatric research, v. 79, n. 4, p. 522-527, 2016.

GEORGE, Valerie A.; MORGANSTEIN, Andrea. Effect of moderate intensity exercise on acute energy intake in normal and overweight females. Appetite, v. 40, n. 1, p. 4346, 2003.

ISMAIL, Irfan et al. A systematic review and meta-analysis of the effect of aerobic vs. resistance exercise training on visceral fat. Obesity reviews, v. 13, n. 1, p. 68-91, 2012 
KOHRT, Wendy $M$. et al. Insulin resistance in aging is related to abdominal obesity. Diabetes, v. 42, n. 2, p. 273-281, 1993.

LEE, Man-Gyoon et al. Effects of high-intensity exercise training on body composition, abdominal fat loss, and cardiorespiratory fitness in middle-aged Korean females. Applied Physiology, Nutrition, and Metabolism, v. 37, n. 6, p. 1019-1027, 2012.

LEMMER, JEFFREY T. et al. Effect of strength training on resting metabolic rate and physical activity: age and gender comparisons. Medicine and science in sports and exercise, v. 33, n. 4, p. 532-541, 2001.

ODA, Kanae et al. Relationship between peak oxygen uptake and regional body composition in Japanese subjects. Journal of Sport and Health Science, v. 3, n. 3, p. 233-238, 2014.

OGDEN, Cynthia L. et al. Prevalence of overweight and obesity in the United States, 1999-2004. Jama, v. 295, n. 13, p. 1549-1555, 2006.

O'HAGAN, Ciara; DE VITO, Giuseppe; BOREHAM, Colin AG. Exercise prescription in the treatment of type 2 diabetes mellitus. Sports Medicine, v. 43, n. 1, p. 39-49, 2013.

OLSON, Thomas P. et al. Changes in inflammatory biomarkers following one-year of moderate resistance training in overweight women. International journal of obesity, v. 31, n. 6, p. 996-1003, 2007.

PHILLIPS, Stuart M.; WINETT, Richard A. Uncomplicated resistance training and health-related outcomes: evidence for a public health mandate. Current sports medicine reports, v. 9, n. 4, p. 208, 2010.

POLLOCK, Michael L. et al. Resistance exercise in individuals with and without cardiovascular disease: benefits, rationale, safety, and prescription an advisory from the committee on exercise, rehabilitation, and prevention, council on clinical cardiology, American Heart Association. Circulation, v. 101, n. 7, p. 828-833, 2000. 
SCHRANZ, Natasha; TOMKINSON, Grant; OLDS, Tim. What is the effect of resistance training on the strength, body composition and psychosocial status of overweight and obese children and adolescents? A systematic review and meta-analysis. Sports Medicine, v. 43, n. 9, p. 893-907, 2013.

SCHWINGSHACKL, Lukas et al. Impact of different training modalities on anthropometric and metabolic characteristics in overweight/obese subjects: a systematic review and network meta-analysis. PloS one, v. 8, n. 12, p. e82853, 2013.

SIGAL, Ronald J. et al. Effects of aerobic training, resistance training, or both on glycemic control in type 2 diabetes: a randomized trial. Annals of internal medicine, v. 147, n. 6, p. 357-369, 2007.

STRASSER, Barbara; SIEBERT, Uwe; SCHOBERSBERGER, Wolfgang. Resistance training in the treatment of the metabolic syndrome. Sports medicine, v. 40, n. 5, p. 397-415, 2010.

TJONNA, Arnt Erik et al. CLINICAL PERSPECTIVE. Circulation, v. 118, n. 4, p. 346354, 2008.

WHALEY, M. M.; PH, Brubaker. Otto RM: ACSM's Guidelines for Exercise Testing and Prescription. 2006.

WILLIAMS, Cameron B. et al. Changes in mechanisms proposed to mediate fat loss following an acute bout of high-intensity interval and endurance exercise. Applied Physiology, Nutrition, and Metabolism, v. 38, n. 12, p. 1236-1244, 2013.

Enviado: Agosto, 2020.

Aprovado: Setembro, 2020. 